\title{
Reappraisal of $H E R 2$ status in the spectrum of advanced urothelial carcinoma: a need of guidelines for treatment eligibility
}

\author{
Anissa Moktefi ${ }^{1}$ - Damien Pouessel ${ }^{2,3} \cdot$ Jing $\mathrm{Liu}^{4,5,6} \cdot$ Nanor Sirab $^{2} \cdot$ Pascale Maille $^{2} \cdot$ Pascale Soyeux $^{2}$. \\ Christiane Copie Bergman $^{1} \cdot$ Marie Luce Auriault ${ }^{7} \cdot$ Dimitri Vordos $^{2,8} \cdot$ Alexandre de la Taille $^{2,8} \cdot$ Stéphane Culine ${ }^{9,10}$. \\ Yves Allory ${ }^{2,11}$
}

Received: 27 July 2017 / Revised: 29 December 2017 / Accepted: 30 December 2017 / Published online: 21 February 2018

(c) United States \& Canadian Academy of Pathology 2018

\begin{abstract}
Although human epidermal growth factor receptor 2 (HER2) may represent a therapeutic target, its evaluation in urothelial carcinoma of the bladder does not rely on a standardized scoring system by immunohistochemistry or fluorescent in situ hybridization (FISH), as reflected by various methodology in the literature and clinical trials. Our aim was to improve and standardize HER2 amplification detection in bladder cancer. We assessed immunohistochemical criteria derived from 2013 American Society of Clinical Oncology (ASCO)/College of American Pathologists (CAPs) guidelines for breast cancer and investigated intratumoral heterogeneity in a retrospective multicentric cohort of 188 patients with locally advanced urothelial carcinoma of the bladder. Immunohistochemistry was performed on 178 primary tumors and 126 lymph node metastases, eligible cases (moderate/strong, complete/incomplete membrane staining) were assessed by FISH. HER2 overexpression was more frequent with 2013 ASCO/CAP than 2007 ASCO/CAP guidelines $(p<0.0001)$. The rate of positive HER2 FISH was similar between primary tumor and lymph node metastases (8\%). Among positive FISH cases, $48 \%$ were associated with moderate/strong incomplete membrane staining that were not scored eligible for FISH by 2007 ASCO/CAP criteria. Among 3+ immunohistochemistry score cases, $67 \%$ were associated with HER2-positive FISH. Concordance between primary tumors and matched lymph node metastases was moderate for immunohistochemistry $(\kappa=0.54$ (CI $95 \%$, $0.41-0.67)$ ) and FISH $(\kappa=0.50$ (CI 95\%, 0.20-0.79)). HER2-positive FISH was more frequent in micropapillary carcinomas $(12 \%)$ and carcinoma with squamous differentiation $(11 \%)$ than in pure conventional carcinoma (6\%). Intratumoral heterogeneity for HER2 immunohistochemistry was observed in $7 \%$ primary tumor and $6 \%$ lymph node metastases; $24 \%$ positive HER 2 FISH presented intratumoral heterogeneity. Our study suggests that HER2 evaluation should include an immunohistochemistry screening step with eligibility for FISH including incomplete/complete and moderate/ strong membrane staining. Spatial or temporal intratumoral heterogeneity prompts to perform evaluation on both tumor and lymph node, and for each histological variant observed.
\end{abstract}

\section{Introduction}

Urothelial carcinoma of the bladder is among the most frequent cancer in men in Europe and North America (fourth cancer in men and ninth in women), and muscle-invasive bladder cancer remains an aggressive disease. The search for

Electronic supplementary material The online version of this article (https://doi.org/10.1038/s41379-018-0023-9) contains supplementary material, which is available to authorized users.

Yves Allory

yves.allory@curie.fr

Extended author information available on the last page of the article therapeutic targets and associated markers predictive of response is of critical importance. Among those potential targets, human epidermal growth factor receptor 2 (HER2) gene encodes for a transmembrane $185 \mathrm{kDa}$ receptor tyrosine kinase protein, and its amplification is the primary mechanism for protein overexpression [1] being reported in $0-25 \%$ urothelial carcinoma of the bladder [2-17].

Although initial studies reported encouraging results, a randomized phase II trial failed to reach the required number of patients and was not able to confirm the effectiveness of trastuzumab (a humanized monoclonal antibody raised against HER2) in advanced or metastatic urothelial carcinoma [18]. This trial failure pointed out the need to screen a huge number of patients to identify those with HER2 amplified 
tumors, and the need of an accurate HER2 amplification rate for power calculation to design clinical trials. As fluorescent in situ hybridization (FISH) technology remains an expensive and time-consuming approach, an immunohistochemical step for screening is applied most frequently for breast [1] and gastric cancers [19]. Assessment of HER2 status relies on a standardized scoring system using initial immunohistochemistry and FISH for confirmation.

In the absence of urothelial carcinoma standards, HER2 immunohistochemistry in urothelial carcinoma of the bladder was scored according to breast cancer 2007 American Society of Clinical Oncology (ASCO)/College of American Pathologists (CAPs) guidelines in the majority of studies with complete membranous staining required for $2+$ and $3+$ immunohistochemistry scores [9-13, 15, 20, 21]. This approach was associated with significant false positive (9.5-52\%) and negative rates (23-38.5\%) suggesting an inadequate immunohistochemistry scoring system [13, 15, $17,21,22]$. Interestingly, guidelines for breast cancer were revised in 2013 [23] to take into account incompletely immunoreactive membranes ("U" shaped), expanding the number of cases with a $2+$ score submitted to FISH assay, and it is unknown whether this new scoring system might be effective in urothelial carcinoma. Overall, this lack of standardization of HER2 testing in urothelial carcinoma of the bladder may account for the variable frequency reported for HER2 overexpression and HER2 amplification, and also impacts the inclusion in clinical trial and therapeutic efficacy evaluation [18, 24-26].

Using a cohort of locally advanced urothelial carcinoma of the bladder treated with radical cystectomy and adjuvant chemotherapy, we assessed immunohistochemical criteria derived from 2013 ASCO/CAP guidelines and investigated intratumoral heterogeneity to improve and standardize HER2 amplification detection in bladder cancer.

\section{Patients and methods}

\section{Study population}

Our retrospective multicentric cohort including 226 consecutive patients with locally advanced urothelial carcinoma of the bladder treated with radical cystectomy and pelvic lymph node dissection followed by an adjuvant platinum-based chemotherapy regimen has been described previously [27].

\section{Tissue samples and pathologic review}

Paraffin-embedded tumor samples were available for 188 patients with 116 paired primary tumors and matched lymph node metastases. Pathological review according to the WHO 2016 classification and TNM 2009 (7th edition) was performed by two pathologists (YA and AM) [28, 29].

\section{Immunohistochemistry}

Immunohistochemical staining was performed on 4- $\mu$ m thick formalin-fixed paraffin-embedded whole tissue sections using standardized automated methodology (Bond; Leica Menarini, Nanterre, France). The deparaffinized sections were stained for HER2 (A0485, Dako, Glostrup, Denmark) after heat-induced antigen retrieval (dilution 1/ 500, pH6). The evaluation was carried out only on the invasive component of the tumor. External controls (absent, weak, moderate and strong HER2 membrane staining on breast carcinoma samples) were included in the experiments.

In order to optimize the immunohistochemistry scoring procedure for bladder tissue, the following parameters were considered: percentage of immunoreactive tumor cells for each intensity of reactivity (classified as "absent", "weak", "moderate", or "strong") and degree of membranous reactivity (complete or incomplete). Finally, HER2 immunohistochemistry scores were assessed according to 2007 ASCO/CAP [2] and 2013 ASCO/CAP guidelines [23] (Supplementary table 1). HER2 intratumoral heterogeneity for HER2 immunohistochemistry test was defined as two spatially distinct tumor areas, one with negative/weak staining, and the other ( $>10 \%$ tumor cells) with moderate/ strong intensity.

\section{Dual probe FISH analysis of HER2}

All samples presenting at least $10 \%$ of cells with moderate or strong membranous staining (complete or incomplete) were evaluated by FISH. Fifty-nine primary tumors and 51 lymph node metastases were eligible for FISH analysis. Eight primary tumors and 18 lymph node metastases could not be assessed due to technical difficulties with the FISH analysis or to insufficient tumor material. FISH was performed on $3 \mu \mathrm{m}$ formalin-fixed paraffin-embedded whole tumor tissue sections using HER2 FISH pharmDx kit (Dako, K533121).

Slides were analyzed with a Zeiss AxioImager Z1 (Zeiss, Göttingen, Germany) fluorescence microscope equipped with microscope-specific double filters (XF53; Omega Optical, Brattleboro, VT) suitable for the fluorescein isothiocyanate and Texas red-labeled signal probes. Images were captured with a $\times 40$ objective using a Hamamatsu digital camera attached to the fluorescence microscope and Visilog 6.9 software (FEI, Les Ullis, France).

Slides were analyzed with a $\times 100$ oil immersion objective. The numbers of chromosome 17 centromere (CEP17) and HER2 signals were counted in 40 
Table 1 Clinicopathological features of our 188 patients with advanced urothelial bladder cancer treated with adjuvant chemotherapy

\begin{tabular}{|c|c|}
\hline Variable & $N(\%)$ or variable unit \\
\hline Median age (years) at cystectomy (range) & $62(35-79)$ \\
\hline \multicolumn{2}{|l|}{ Gender } \\
\hline Female & $31(17)$ \\
\hline Male & $157(83)$ \\
\hline \multicolumn{2}{|l|}{ pT stage, $N(\%)$} \\
\hline T0 & $7(4)$ \\
\hline Tis & $1(1)$ \\
\hline $\mathrm{Ta}$ & $1(1)$ \\
\hline $\mathrm{T} 1$ & $4(2)$ \\
\hline $\mathrm{T} 2$ & $25(13)$ \\
\hline $\mathrm{T} 3$ & $94(50)$ \\
\hline $\mathrm{T} 4$ & $56(29)$ \\
\hline \multicolumn{2}{|l|}{ pN stage, $N(\%)$} \\
\hline $\mathrm{Nx}$ & $18(9)$ \\
\hline No & $28(15)$ \\
\hline N1 & $45(24)$ \\
\hline $\mathrm{N} 2$ & $96(51)$ \\
\hline N3 & $1(1)$ \\
\hline \multicolumn{2}{|l|}{ Treatment } \\
\hline \multicolumn{2}{|l|}{ Adjuvant chemotherapy regimen } \\
\hline $\mathrm{G}$ & $1(1)$ \\
\hline GC & $131(69)$ \\
\hline $\mathrm{GCb}$ & $45(24)$ \\
\hline MVAC & $9(5)$ \\
\hline Other & $2(1)$ \\
\hline \multicolumn{2}{|c|}{ Histological variant of primary tumors $(N=178)^{*}$} \\
\hline Pure conventional urothelial carcinoma & $68(38)$ \\
\hline \multicolumn{2}{|c|}{ Urothelial carcinoma with divergent differentiation } \\
\hline Squamous differentiation & $44(25)$ \\
\hline Glandular differentiation & $4(2)$ \\
\hline Micropapillary carcinoma & $50(28)$ \\
\hline Pure micropapillary carcinoma & 20 \\
\hline Mixte micropapillary carcinoma & 30 \\
\hline Plasmacytoid/single-cell carcinoma & $13(7)$ \\
\hline Sarcomatoid carcinoma & $23(13)$ \\
\hline Giant cell & $1(1)$ \\
\hline Microcystic & $1(1)$ \\
\hline
\end{tabular}

$A$ doxorubicin, $G$ gemcitabine, $C$ cisplatin, $C b$ carboplatin, $M$ methotrexate, $V$ vinblastine

*Sum of percentages above $100 \%$ due to tumor heterogeneity

non-overlapping nuclei. Only cells on which at least two CEP17 reference probe signals could be identified were included. For each case, the average HER2 copy number, the average number of CEP17, and the ratio of HER2 signals to CEP17 signals were calculated. According to 2013
ASCO/CAP HER2 test guidelines, a HER2/CEP17 ratio $\geq$ 2.0 was reported as positive. When the HER2/CEP17 ratio was $<2.0$, an average $H E R 2$ copy number $\geq 6$ was reported as positive, a copy number $<4.0$ was reported as negative, and a copy number in the range of 4.0-5.9 was reported as equivocal. Chromosome 17 polysomy was defined as $\geq 3$ CEP17 signals per cell. For heterogeneous cases, histological variant, HER2 immunohistochemistry scores, and HER2 FISH status were reported for each component.

\section{Statistical analysis}

Frequencies and proportions were reported for categorical variables. The Chi2 Mc Nemar test measured the difference in immunohistochemistry results using 2007 and 2013 ASCO/CAP guidelines. A $p$-value $<0.05$ was required for statistical significance. Cohen's kappa (with linear weighting) assessed the concordance of HER2 status of the gene and protein between primary tumor and matched lymph node metastases.

Overall survival was assessed from the date of cystectomy to the date of death. The Kaplan-Meier method was used to visualize the survival distribution. The Cox proportional hazards model was used to estimate hazard ratio (HR) and test the statistical significance. The test was two-sided, and $p$-value $<0.05$ was considered significant. The survival analysis was performed under $\mathrm{R}$ version 3.4.2.

\section{Results}

\section{Patient characteristics}

The clinicopathologic features of our cohort of 188 patients with advanced urothelial carcinoma of the bladder treated with radical cystectomy and adjuvant chemotherapy are listed in Table 1. Eighty percent of patients demonstrated pT3 or pT4 stage. Seventy-five percent of patients presented with lymph node metastases. Gemcitabine with cisplatin, gemcitabine with carboplatin, and methotrexate, vinblastine, doxorubicin, and cisplatin (MVAC) regimens were delivered in 131 (69\%), 45 (24\%), and 9 patients (5\%), respectively. Primary tumors histological material was available for 178 patients. The most frequent histological variants were pure conventional urothelial carcinoma (38\%), micropapillary carcinoma (28\%), and urothelial carcinoma with squamous differentiation $(25 \%)$.

\section{HER2 protein expression and eligibility to HER2 FISH}

Evaluation of HER2 protein expression was performed by immunohistochemistry (Fig. 1, 2). We scored HER2 protein 
expression according to 2007 ASCO/CAP guidelines and observed a majority of score $1+$ cases (103 primary tumors $(58 \%)$ and 81 lymph node metastases $(64 \%)$ ) with only score 2 or 3 in 18 primary tumors (10\%) and 15 lymph node metastases (12\%). With the 2013 ASCO/CAP guidelines, the majority of cases presented a score of $2+(93$ primary tumors (52\%) and 73 lymph node metastases (58\%)) and we noticed an increase of the number of cases with the score $3+(7$ primary tumors $(4 \%)$ and 4 lymph node metastases (3\%)). Overall, the number of cases with HER2 protein overexpression $(2+$ or $3+$ immunohistochemistry score) was statistically more important according to 2013 ASCO/CAP guidelines than the 2007 ASCO/CAP criteria $(p<0.0001$, chi2 Mc Nemar test).

In this study, we assessed HER2 FISH status focusing in cases exhibiting a moderate or intense, complete or incomplete membrane staining in $\geq 10 \%$ of tumors cells. Four cases presented intense complete circumferential intense staining in $<10 \%$ of tumor cells, but all were associated with a moderate staining justifying eligibility for FISH. With these criteria, the number of eligible cases for HER2 FISH was intermediate between the 2013 and the 2007 ASCO/CAP guidelines (59 primary tumors (33\%) and 51 lymph node metastases (40\%)).

\section{Correlation between HER2 gene status and HER2 protein expression}

HER 2 status could be determined by FISH in 51 primary tumors and 33 lymph node metastases (Fig. 1). HER2 status was positive in 15 primary tumors $(30 \%)$ and 10 lymph node metastases $(30 \%)$, equivocal in 16 primary tumors (31\%) and 11 lymph node metastases (33\%), and negative in 20 primary tumors (39\%) and 12 lymph node metastases (37\%) (Supplementary Table 2 and Fig. 3). We compared HER2 FISH status and HER2 immunohistochemistry score according to 2007 and 2013 ASCO/CAP guidelines (Table 2a and Supplementary table 3), 10 primary tumors and 4 lymph node metastases among them presenting heterogeneous HER2 immunohistochemistry scores. Among the 25 positive $H E R 2$ FISH cases, eight primary tumors and four lymph node metastases had a score $1+$, four primary tumors and five lymph node metastases score $2+$ and three primary tumors and one lymph node metastases score $3+$, in the 2007 ASCO/CAP system. The 12 cases with $1+$ score exhibited incomplete membrane staining. With the more recent 2013 ASCO/CAP criteria, 12 primary tumors and 7 lymph node metastases showed $2+$ staining and 3 primary tumors and 3 lymph node metastases had $3+$ staining. Thirty primary tumors and 14 lymph node metastases were HER2 FISH negative, none of them associated with $3+$ staining in the 2007 and 2013 ASCO/CAP guidelines. Among 29 equivocal (16 primary tumors and 13

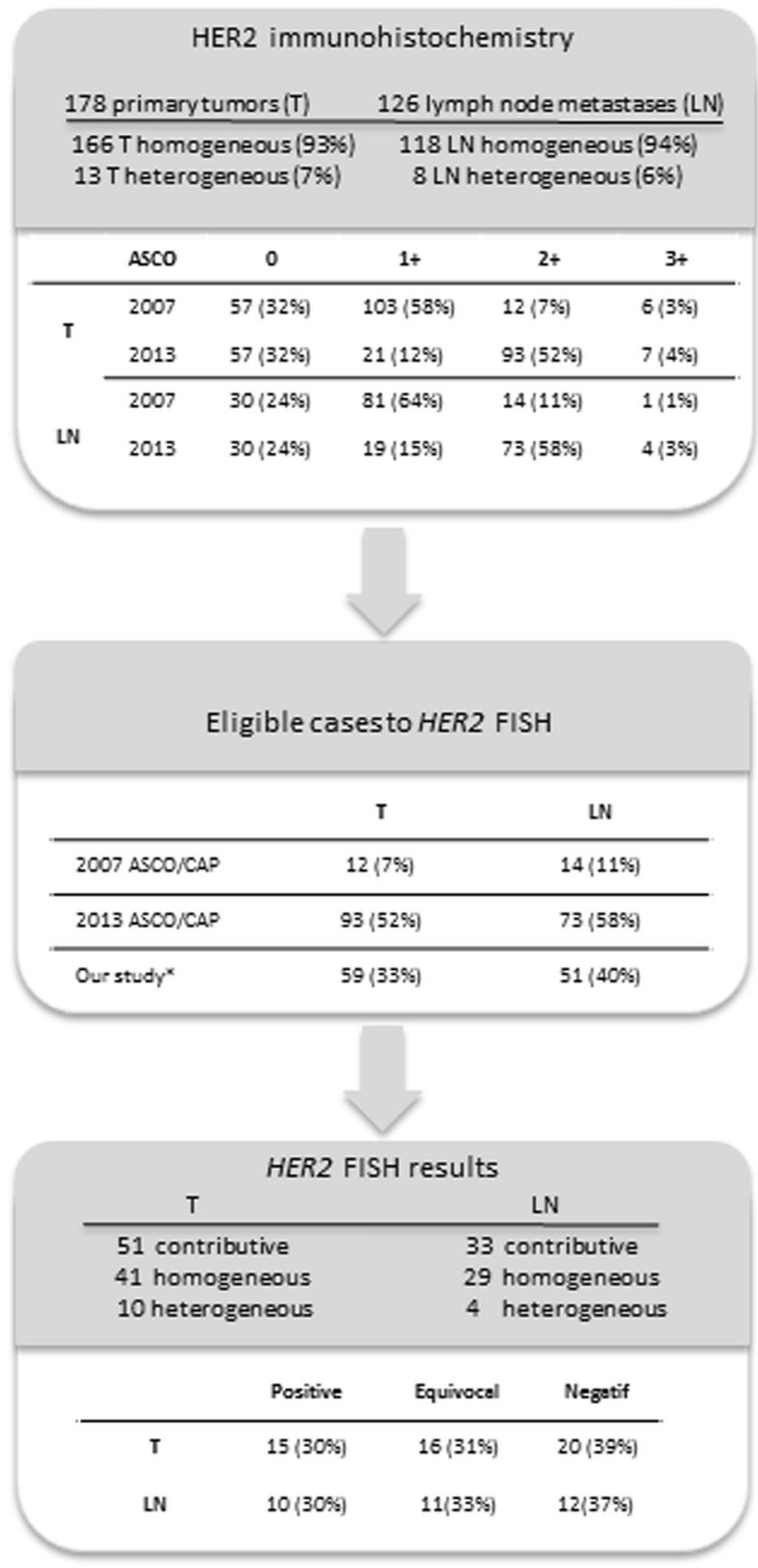

Fig. 1 Flowchart showing the three-step approach for HER2 assessment in our cohort of locally advanced urothelial carcinoma of the bladder. * Eligible cases to FISH in our study: moderate or strong, complete or incomplete HER2 membrane staining in $\geq 10 \%$ of tumor cells. ASCO/CAP American Society of Clinical Oncology/College of American Pathologists, FISH fluorescent in situ hybridization, HER2 human epidermal growth factor receptor 2, LN lymph node metastases, $\mathrm{T}$ primary tumor

lymph node metastases) and 44 negative (30 primary tumors and 14 lymph node metastases) HER2 FISH cases, $24(83 \%)$ and $23(52 \%)$ cases showed polysomy, respectively. Overall, 54\% primary tumors and $70 \%$ lymph node metastases presented polysomy with a mean CEP17 copy number $3.8( \pm 0.9)$, and $3.7( \pm 0.6)$, respectively. 


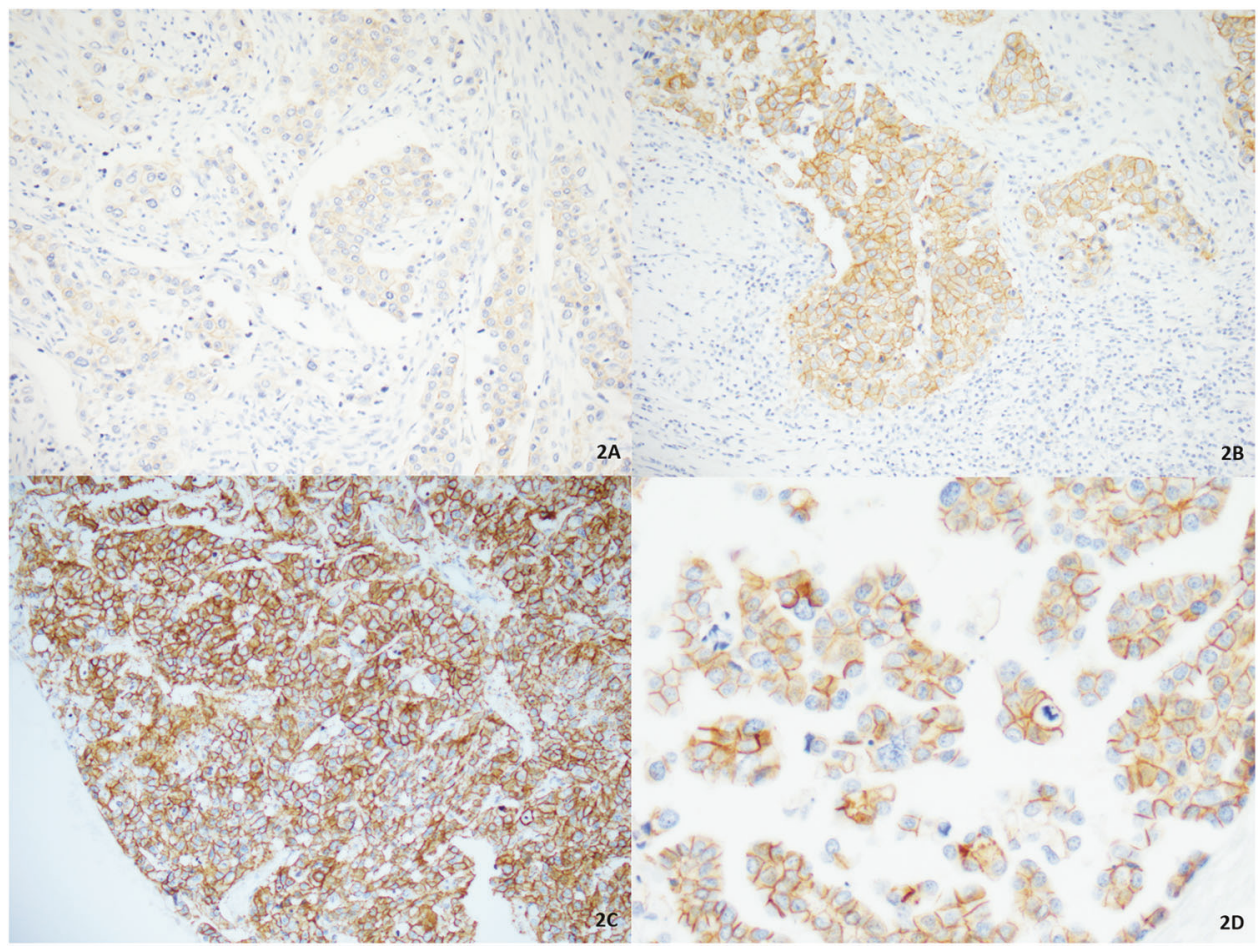

Fig. 2 HER2 expression in urothelial carcinoma by immunohistochemistry. a Weak complete membrane staining: score 1+ according to 2007 ASCO/CAP guidelines and score 2+ according to 2013 ASCO/CAP guidelines $(\times 200)$. b Moderate complete membrane staining: score $2+$ according to 2007 and 2013 ASCO/CAP guidelines $(\times 200)$. c Strong complete membrane staining: score $3+$ according to 2007 and 2013 ASCO/CAP guidelines $(\times 200)$. d Moderate

\section{Comparison of HER2 immunohistochemistry scores and HER2 FISH status between primary tumors and matched lymph node metastases}

The HER2 immunohistochemistry score was evaluated for 116 paired primary tumors and matched lymph node metastases (Table 2b). As 2007 ASCO/CAP was less sensitive than the 2013 system, we focus thereafter on 2013 ASCO/CAP. Seventy-eight (67\%) lymph node metastases exhibited the same score than corresponding primary tumor. Twenty-three (20\%) lymph node metastases showed a higher HER2 immunohistochemistry score, whereas 15 cases (13\%) had a lower HER2 immunohistochemistry score than the matched primary tumor $(\kappa=0.54$ (confidence interval (CI) 95\%, $0.41-0.67)$ ). We evaluated HER2 FISH status in 22 paired primary tumor and matched lymph node metastases (Table 2b). Fourteen (63\%) cases exhibited concordant HER2 FISH status between primary tumor and matched lymph node metastases $(\kappa=0.50$ (CI 95\%, $0.20-0.79)$ ), whereas one primary tumor positive for $H E R 2$ FISH assay was associated with a negative HER2 incomplete ("U" shape) membrane staining in a micropapillary urothelial carcinoma. This case would be scored $1+$ according to 2007 ASCO/CAP guidelines and 2+ with 2013 ASCO/CAP guidelines $(\times 400)$. ASCO/CAP American Society of Clinical Oncology/College of American Pathologists, HER2 human epidermal growth factor receptor 2

FISH lymph node metastases and conversely one negative FISH HER2 primary tumor corresponded to positive HER2 FISH lymph node metastases.

\section{Association between HER2 gene status, HER2 protein expression, and histological variant}

HER2 gene status and HER2 protein expression were studied according to the histological variant (Table 3). Cases with moderate/strong and incomplete/complete membrane staining represented $22 \%, 27 \%$, and $23 \%$ of pure conventional urothelial carcinoma, urothelial carcinoma with squamous differentiation and plasmacytoid carcinoma, respectively. Pure micropapillary carcinoma displayed the highest proportion of eligible cases (15 primary tumors $(75 \%)$ ), whereas sarcomatoid carcinoma presented the lowest (3 primary tumors (13\%)). Among 50 micropapillary urothelial carcinoma, 6 cases showed positive HER2 FISH (12\%). HER2 FISH was positive in $6 \%$ pure conventional, $9 \%$ sarcomatoid, and $11 \%$ divergent differentiation with squamous feature urothelial carcinoma. None plasmacytoid carcinoma was positive. 


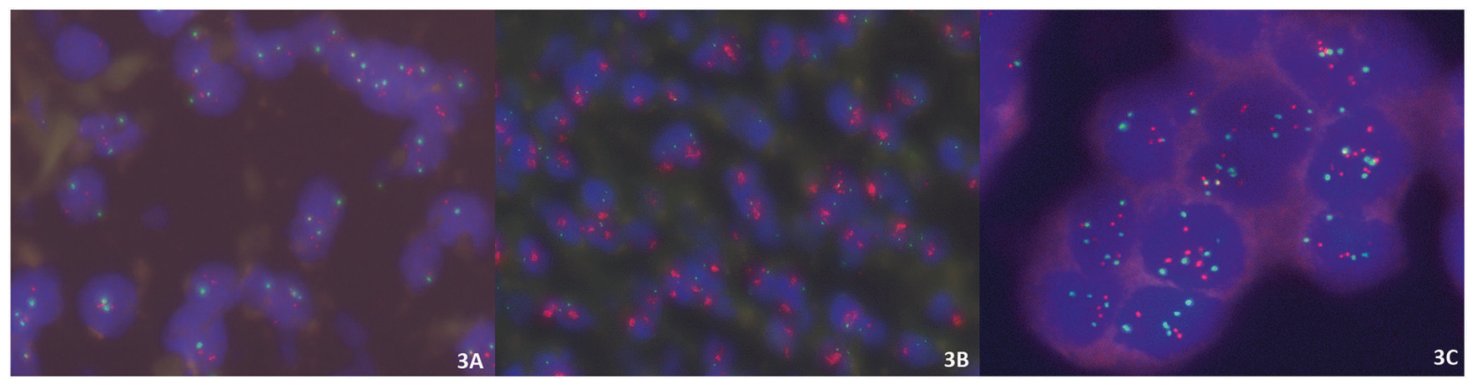

Fig. 3 HER 2 gene status in urothelial carcinoma. Red probe indicates HER 2 and green probe indicates chromosome 17 centromere. a Negative HER2 FISH assay (×400). b Amplified/positive HER2 FISH

\section{HER2 heterogeneity}

We identified HER2 protein expression heterogeneity in 20 patients (13 primary tumors $(7 \%)$ and 7 lymph node metastases $(6 \%)$ ) with 2 paired primary tumors and lymph node metastases both exhibiting HER2 immunohistochemistry score heterogeneity (Table 4 and Fig. 4). Heterogeneous HER2 protein expression corresponded to different histological variants in 13 cases among which 10 cases presented a combination of conventional and micropapillary urothelial carcinomas. HER2 FISH status could not be assessed for six heterogeneous cases. Among the other 14 cases with HER 2 protein heterogeneity, we observed 10 cases with heterogeneous HER2 FISH status: 5 negative/ positive cases, 4 negative/equivocal cases, and 1 equivocal/ positive case. Four cases with HER 2 protein heterogeneity demonstrated a homogeneous HER2 FISH status (three cases with negative and one case with equivocal HER2 FISH). HER2 FISH assay was available in lymph node metastases for three primary tumors with a heterogeneous negative/positive HER2 FISH assay. One lymph node metastases presented a positive HER2 FISH, another showed an equivocal HER2 FISH and the third one had a negative HER2 FISH.

\section{Prognostic impact of HER2 status}

Ten-year overall survival was not different between patients with HER2 negative primary tumor and lymph node metastases versus patients with $H E R 2$ positive/equivocal primary tumor or lymph node metastases $(\mathrm{HR}=1.3 ; 95 \%$ CI $(0.8-2.2), p=0.24)$, although a trend for a worse outcome was associated with the positive/equivocal cases (Supplementary Figure 1).

\section{Discussion}

HER2 amplification detection is pivotal for patient eligibility to targeted therapy but various methodologies for assay with clusters of $H E R 2$ gene signals $(\times 400)$. c Polysomy for HER2 $(\times 400)$. FISH fluorescent in situ hybridization, HER2 human epidermal growth factor receptor 2

HER2 evaluation have been used in urothelial carcinoma of the bladder (immunohistochemistry screening or not, distinct criteria for $H E R 2$ amplification) with a lack of standard criteria (see studies listed in supplementary table 4). In our study, we provided strong evidence supporting the use of immunohistochemistry criteria derived from updated breast cancer ASCO/CAP guidelines to optimize HER2 amplification detection in urothelial carcinoma of the bladder. This methodology allowed us a reappraisal of HER2 amplification in urothelial cancer in relationships with histological variants and intratumoral heterogeneity.

HER2 immunohistochemistry scoring is used to screen tumors potentially with HER2 amplification, whereas FISH assay is considered as the gold standard. For breast and gastric cancers, positivity criteria have been optimized to provide a sensitive method. The latest 2013 ASCO/CAP guidelines for HER2 evaluation in breast cancer considers (circumferential membrane staining that is incomplete and/ or weak/moderate and within $>10 \%$ of tumor cells) as $2+$ immunohistochemistry score, whereas incomplete membrane staining was scored $1+$ only according to 2007 ASCO/CAP guidelines. Similarly, incomplete moderate and strong membrane staining are, respectively, scored $2+$ and 3+ in HER2 scoring system for gastric cancer [19]. Our study was performed on locally advanced urothelial carcinoma of the bladder, which reflects better metastatic disease than earlier stages. We performed HER2 FISH assay for cases presenting moderate/strong complete or incomplete membrane staining in $>10 \%$ of cells, excluding only cases with weak complete/incomplete staining, and identified positive HER 2 FISH in $~ 8 \%$ cases. With the 2007 ASCO/CAP system scoring moderate incomplete staining as $1+, 12$ positive HER2 FISH cases would not have been assessed by FISH and diagnosed as not amplified. Unlike Lae et al., we observed also that complete moderate staining (2+ according to 2007 and 2013 ASCO/ CAP guidelines) can be associated with positive HER2 FISH [12]. Among nine cases with 3+ immunohistochemistry score according to 2013 ASCO/CAP guidelines, only six cases displayed positive HER2 FISH, 
Table 2 A) Comparison between HER2 immunohistochemistry score and HER2 FISH status in primary tumors and lymph node metastases, B) Comparison of HER2 status between primary tumors and matched lymph node metastases

\begin{tabular}{|c|c|c|c|c|}
\hline \multicolumn{5}{|l|}{ A) } \\
\hline \multirow[t]{2}{*}{ FISH } & \multicolumn{4}{|c|}{ HER2 IHC test according to 2007 ASCO/CAP guidelines } \\
\hline & $0(N=4)$ & $1+(N=65)$ & $2+(N=23)$ & $3+(N=6)$ \\
\hline Positive $(N=25)$ & 0 & $12(18 \%)$ & $9(39 \%)$ & $4(67 \%)$ \\
\hline Equivocal $(N=29)$ & 0 & $22(34 \%)$ & $5(22 \%)$ & $2(33 \%)$ \\
\hline $\operatorname{Polysomy}(N=24)$ & 0 & 19 & 3 & 2 \\
\hline $\operatorname{Negative}(N=44)$ & $4(100 \%)$ & $31(48 \%)$ & $9(39 \%)$ & 0 \\
\hline $\operatorname{Polysomy}(N=23)$ & 2 & 15 & 6 & 0 \\
\hline \multirow[t]{2}{*}{ FISH } & \multicolumn{4}{|c|}{ HER2 IHC test according to 2013 ASCO/CAP guidelines } \\
\hline & $0(N=4)$ & $1+(N=4)$ & $2+(N=81)$ & $3+(N=9)$ \\
\hline Positive $(N=25)$ & 0 & 0 & $19(23 \%)$ & $6(67 \%)$ \\
\hline Equivocal $(N=29)$ & 0 & $1(25 \%)$ & $25(31 \%)$ & $3(33 \%)$ \\
\hline $\operatorname{Polysomy}(N=24)$ & 0 & 1 & 20 & 3 \\
\hline $\operatorname{Negative}(N=44)$ & $4(100 \%)$ & $3(75 \%)$ & $37(46 \%)$ & 0 \\
\hline $\operatorname{Polysomy}(N=23)$ & 2 & 1 & 20 & 0 \\
\hline
\end{tabular}

B)

\begin{tabular}{|c|c|c|c|c|c|c|}
\hline & \multirow[b]{2}{*}{$\begin{array}{l}\text { IHC } \\
2013 \mathrm{ASCO} / \mathrm{CAP}\end{array}$} & \multicolumn{5}{|c|}{ Lymph node metastases } \\
\hline & & 0 & $1+$ & $2+$ & $3+$ & Total \\
\hline \multirow[t]{10}{*}{ Primary tumor } & 0 & 20 & 8 & 3 & 0 & 31 \\
\hline & $1+$ & 0 & 8 & 9 & 0 & 17 \\
\hline & $2+$ & 9 & 3 & 49 & 3 & 64 \\
\hline & $3+$ & 0 & 0 & 3 & 1 & 4 \\
\hline & Total & 29 & 19 & 64 & 4 & 116 \\
\hline & FISH & Positive & Equivocal & \multicolumn{2}{|l|}{ Negative } & Total \\
\hline & Positive & 7 & 1 & \multicolumn{2}{|l|}{$1^{\mathrm{a}}$} & 9 \\
\hline & Equivocal & 0 & 4 & \multicolumn{2}{|l|}{1} & 5 \\
\hline & Negative & $1^{\mathrm{b}}$ & 4 & \multicolumn{2}{|l|}{3} & 8 \\
\hline & Total & 8 & 9 & \multicolumn{2}{|l|}{5} & 22 \\
\hline
\end{tabular}

\footnotetext{
${ }^{a}$ Heterogeneous primary tumor with homogeneous lymph node metastases

${ }^{\mathrm{b}}$ HER 2 copy number per cell $=3.93$ in primary tumor

ASCO/CAP: American Society of Clinical Oncology/College of American Pathologists, FISH: fluorescent in situ hybridization, HER2: Human Epidermal growth factor Receptor 2, IHC: immunohistochemistry.
}

whereas the three others showed equivocal HER2 FISH, suggesting that HER2 FISH assay remains required for strong complete membrane staining. "False positive $3+$ immunohistochemistry score" urothelial carcinoma of the bladder have been reported also by Fleischmann et al. (9.5\%) [13] and Schneider et al. (52\%) [21].

The 2013 ASCO/CAP HER2 guidelines for FISH included not only HER2/CEP17 ratio $\geq 2.0$ but also HER2 copy number as positive criteria. In our cohort assessed with the same 2013 guidelines, 20\% of HER2-positive FISH were due to $H E R 2$ polysomy ( $\geq 6$ copies) independently of HER2/CEP17 ratio.
Our HER2 amplification detection strategy derived from the 2013 ASCO/CAP guidelines for breast cancer allowed us to revisit its frequency according to bladder carcinoma histological variants. In our cohort of locally advanced urothelial carcinoma of the bladder, only $38 \%$ were pure conventional urothelial carcinoma underscoring the enrichment of variants. It was reported an overall 53\% incidence rate of bladder cancer variants in a series of 302 patients treated by cystectomy [30]. Similarly, unusual morphological variants represented $40 \%$ cases in a cohort of locally advanced (74\% pT2-T4 stage) high-grade urothelial carcinomas of the renal pelvis [31]. Previous studies 


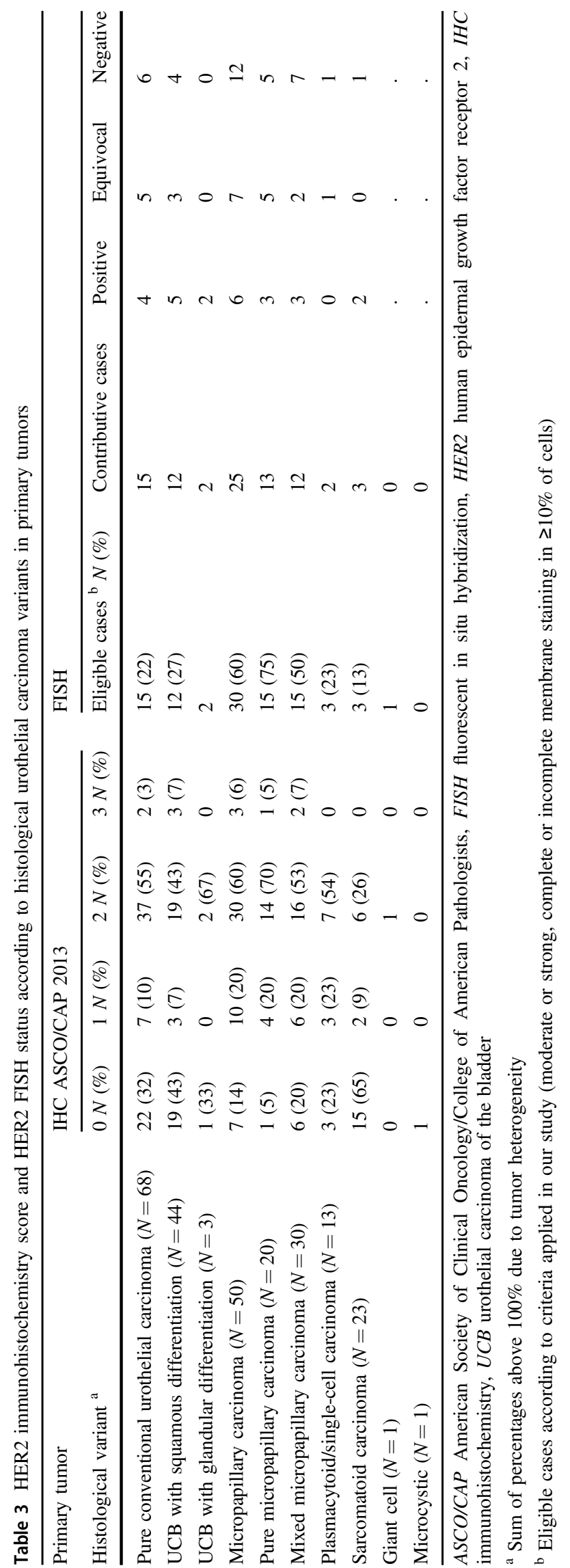

underscored higher HER2 protein overexpression and HER2 amplification rate in micropapillary carcinomas $[20,21]$. According to recent molecular classification of urothelial carcinoma of the bladder, micropapillary carcinoma is considered as luminal type [32], which has been associated also with HER2 protein overexpression [16, 33]. Our results showed $60 \%$ micropapillary carcinomas with HER 2 protein overexpression, but only $12 \%$ showed positive HER2-positive FISH versus $6 \%$ in conventional urothelial carcinoma. These results confirmed another report of $15 \%$ and $9 \%$ of HER2 amplification for micropapillary carcinoma and conventional urothelial carcinoma matched on stage [21]. Hence HER2 protein overexpression in micropapillary carcinomas is linked only partly to HER2 amplification. A high frequency (40\%) of HER2 mutation has been detected in micropapillary urothelial carcinoma [34] without protein overexpression, suggesting that $H E R 2$ mutations are not responsible for the discrepancy between protein overexpression and amplification in micropapillary carcinomas. In our cohort, urothelial carcinoma with squamous differentiation (viewed as basal/squamous-like urothelial carcinoma at molecular level) displayed also a significant rate of positive HER2 FISH (11\%). Unlike Kim et al. who reported that $50 \%$ plasmacytoid carcinoma displayed positive HER2 FISH [35], none of the 13 plasmacytoid/single-cell carcinomas presented positive HER2 FISH. Of note, a comparative genomic hybridization on 25 plasmacytoid urothelial carcinoma revealed gains on chromosome $17 \mathrm{q}$ in $88 \%$ cases but only $14 \%$ showed HER 2 amplification [36].

Intratumoral HER2 heterogeneity has been rarely studied in urothelial carcinoma of the bladder. Lae et al. reported HER 2 protein heterogeneity in $35 \%$ of urothelial carcinoma of the bladder with a $3+$ immunohistochemistry score (1.8\% of cohort) with FISH showing exactly the same heterogeneous pattern with adjacent positive and negative areas [12]. HER2 intratumoral heterogeneity has been shown to be frequent in gastroesophageal [37] and breast cancers $[38,39](5-69 \%$ and 5-40\%, respectively). This wide range might reflect the absence of standardized definition for HER2 heterogeneity. The definition of HER2 heterogeneity in breast cancer has only recently been clarified in 2013 ASCO/CAP guidelines. In our study, HER2 intratumoral heterogeneity for the HER2 immunohistochemistry test was defined as two distinct tumor areas, one with negative/weak staining, and the other $(>10 \%$ tumor area) with moderate/strong intensity and excluded mosaic pattern. Six to $7 \%$ of cases displayed HER2 protein heterogeneity, and HER2 protein heterogeneity was associated with HER 2 FISH heterogeneity in $71 \%$ of cases. Interestingly, heterogeneous cases represent $\sim 1 / 4$ positive HER2 FISH (6/25), suggesting that intratumoral heterogeneity of HER 2 expression may potentially contribute to 
Table 4 Characteristics of primary tumors and matched lymph node metastases with HER2 heterogeneous expression

\begin{tabular}{|c|c|c|c|c|c|}
\hline Case & & Histological variant & $\begin{array}{l}\text { Homogeneity for HER } 2 \\
\text { IHC score }\end{array}$ & $\begin{array}{l}\text { HER2 membrane } \\
\text { staining intensity }\end{array}$ & FISH \\
\hline \multirow[t]{4}{*}{$\# 1$} & $\mathrm{~T}$ & Squamous differentiation & Heterogeneous & Absent & Negative \\
\hline & $\mathrm{T}$ & Micropapillary & & Moderate & Positive \\
\hline & $\mathrm{LN}$ & Conventional & Heterogeneous & Weak & Equivocal \\
\hline & $\mathrm{LN}$ & Micropapillary & & Moderate & Positive \\
\hline \multirow[t]{4}{*}{$\# 2$} & $\mathrm{~T}$ & Conventional & Heterogeneous & Weak & Negative \\
\hline & $\mathrm{T}$ & Glandular differentiation & & Moderate & Positive \\
\hline & $\mathrm{LN}$ & Conventional & Homogeneous & Moderate & Equivocal \\
\hline & $\mathrm{LN}$ & Micropapillary & & Moderate & Equivocal \\
\hline \multirow[t]{4}{*}{ \#3 } & $\mathrm{T}$ & Conventional & Homogeneous & Moderate & Negative \\
\hline & $\mathrm{T}$ & Micropapillary & & Moderate & Negative \\
\hline & $\mathrm{LN}$ & Conventional & Heterogeneous & Weak & Negative \\
\hline & $\mathrm{LN}$ & Micropapillary & & Moderate & Equivocal \\
\hline \multirow[t]{4}{*}{ \#4 } & $\mathrm{T}$ & Conventional & Heterogeneous & Weak & Negative \\
\hline & $\mathrm{T}$ & Conventional & & Moderate & Equivocal \\
\hline & $\mathrm{LN}$ & Squamous differentiation & Heterogeneous & Weak & $\mathrm{NC}$ \\
\hline & $\mathrm{LN}$ & Squamous differentiation & & Moderate & $\mathrm{NC}$ \\
\hline \multirow[t]{4}{*}{$\# 5$} & $\mathrm{~T}$ & Conventional & Heterogeneous & Weak & Negative \\
\hline & $\mathrm{T}$ & Micropapillary & & Moderate & Negative \\
\hline & $\mathrm{LN}$ & Conventional & Homogeneous & Moderate & Negative \\
\hline & $\mathrm{LN}$ & Micropapillary & & Moderate & Negative \\
\hline \multirow[t]{4}{*}{$\# 6$} & $\mathrm{~T}$ & Conventional & Homogeneous & Weak & $\mathrm{NC}$ \\
\hline & $\mathrm{T}$ & Micropapillary & & Weak & $\mathrm{NC}$ \\
\hline & $\mathrm{LN}$ & Conventional & Heterogeneous & Weak & Negative \\
\hline & $\mathrm{LN}$ & Micropapillary & & Moderate & Positive \\
\hline \multirow[t]{2}{*}{$\# 7$} & $\mathrm{~T}$ & Squamous differentiation & Heterogeneous & Weak & Negative \\
\hline & $\mathrm{T}$ & Micropapillary & & Strong & Positive \\
\hline \multirow[t]{3}{*}{$\# 8$} & $\mathrm{~T}$ & Conventional & Heterogeneous & Weak & Negative \\
\hline & $\mathrm{T}$ & Micropapillary & & Strong & Equivocal \\
\hline & $\mathrm{LN}$ & Micropapillary & Homogeneous & Moderate & $\mathrm{NC}$ \\
\hline \multirow[t]{2}{*}{ \#9 } & $\mathrm{T}$ & Conventional & Heterogeneous & Weak & Negative \\
\hline & $\mathrm{T}$ & Micropapillary & & Moderate & Negative \\
\hline \multirow[t]{2}{*}{$\# 10$} & $\mathrm{~T}$ & Conventional & Heterogeneous & Absent & Negative \\
\hline & $\mathrm{T}$ & Conventional & & Moderate & Negative \\
\hline \multirow[t]{3}{*}{$\# 11$} & $\mathrm{~T}$ & Conventional & Heterogeneous & Absent & Negative \\
\hline & $\mathrm{T}$ & Plasmacytoid & & Moderate & Equivocal \\
\hline & $\mathrm{LN}$ & Conventional & Homogeneous & Moderate & $\mathrm{NC}$ \\
\hline \multirow[t]{4}{*}{$\# 12$} & $\mathrm{~T}$ & Sarcomatoid & Heterogeneous & Absent & Negative \\
\hline & $\mathrm{T}$ & Squamous differentiation & & Moderate & Positive \\
\hline & $\mathrm{LN}$ & Sarcomatoid & Homogeneous & Moderate & Negative \\
\hline & $\mathrm{LN}$ & Squamous differentiation & & Moderate & Negative \\
\hline \multirow[t]{3}{*}{$\# 13$} & $\mathrm{~T}$ & Conventional & Homogeneous & Moderate & Equivocal* \\
\hline & $\mathrm{LN}$ & $\begin{array}{l}\text { Conventional with extra } \\
\text { capsular extension }\end{array}$ & Heterogeneous & Weak & Equivocal $* *$ \\
\hline & $\mathrm{LN}$ & $\begin{array}{l}\text { Conventional carcinoma } \\
\text { within sinuses }\end{array}$ & & Moderate & Equivocal $* * *$ \\
\hline
\end{tabular}

Only cases with at least one sample with contributive FISH are reported

$\# 13$, equivocal HER2/CEP17 ratio $=* 1.62, * * 1.77, * * * 1.96$

FISH fluorescent in situ hybridization, HER2 human epidermal growth factor receptor 2, IHC immunohistochemistry, $L N$ lymph node metastases, $N C$ non-contributive, $\mathrm{T}$ primary tumor 


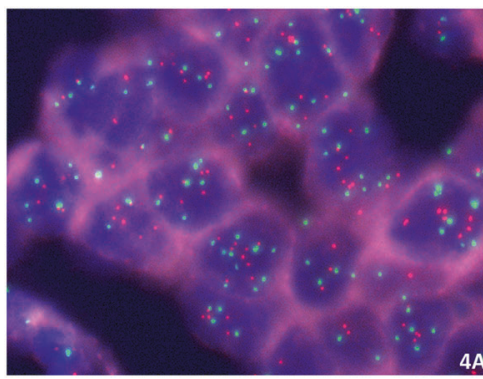

Fig. 4 HER2 heterogeneity in urothelial carcinoma. a, c HER2 FISH (red probe indicates HER2 and green probe indicates chromosome 17 centromere), b HER2 immunohistochemistry. b Showing heterogeneity of staining in an urothelial carcinoma of the bladder. Moderate staining $(2+)$ in micropapillary carcinoma (right side of image) adjacent to conventional carcinoma with faint staining $(1+)$ (left side of

inaccurate assessment of HER2 status. HER2 evaluation should be performed preferentially on cystectomy samples rather than transurethral resection of bladder tumor due to HER2 intratumoral heterogeneity. Pathologists should be aware that HER2 heterogeneity in urothelial carcinoma of the bladder is frequently associated with different coexisting histological variants, and give priority to samples with micropapillary carcinomas for HER2 evaluation. Comparing primary tumor and matched metastatic lymph node, we observed HER2 immunohistochemistry and HER2 FISH score concordance in a majority of paired primary tumors and matched lymph node metastases. However, twenty-three (20\%) lymph nodes showed a higher HER2 immunohistochemistry score than the matched primary tumor suggesting that HER2 should be evaluated systematically on primary tumor and lymph node metastases in urothelial carcinoma of the bladder. Three series investigated HER2 status in lymph node-positive urothelial carcinoma of the bladder. Hansel et al. reported a higher concordance rate $(88 \%)$ for HER2 immunohistochemistry score between primary tumor and matched lymph node metastases [11]. More frequent HER2 overexpression and HER2 amplification in lymph node metastases than in primary tumor have also been reported [13, 40].

There are several limits to our study. We did not assess FISH on the whole cohort but on eligible cases based on immunohistochemistry results. As we did not investigate cases with weak and incomplete membranous staining, we cannot exclude that such staining is occasionally associated to positive HER2 FISH in urothelial carcinoma of the bladder.

The results concerning HER2 FISH status in lymph node metastases should be interpreted with cautiousness because $35 \%$ eligible lymph node metastases could not be assessed due to technical difficulties with the FISH analysis or to insufficient tumor material. Of note, only one noncontributive lymph node metastases corresponded to a positive HER 2 FISH primary tumor. We identified a higher image) $(\times 100)$. a Conventional carcinoma with faint staining was associated with negative HER2 FISH assay (ratio $=1.06$ and polysomy (mean centromere 17 copy/cell $=3.55))(\times 400)$ whereas amplification of HER2 was noted in micropapillary areas with HER2 overexpression c $(\times 400)$. FISH fluorescent in situ hybridization, HER2 human epidermal growth factor receptor 2

rate of equivocal cases (29\%) than in breast cancer $(1.9-9.5 \%)[41,42]$ but it was beyond the scope of the study to perform an additional test to resolve these equivocal results.

Although phase II studies with anti-HER2 therapies (Trastuzumab and Afatinib) [24, 25] demonstrated encouraging results, a randomized phase III study in stage IV urothelial carcinoma of the bladder patients with $2+$ to 3 + immunohistochemistry score and/or Epidermal Growth Factor Receptor expression did not find significant improvements in outcome by the addition of maintenance lapatinib to standard of care [26]. Accurate assessment of HER2 in urothelial carcinoma appears necessary to select properly eligible patients in clinical trials and to have reliable conclusions about anti-HER2 therapy benefits in urothelial carcinoma of the bladder. Recently, Kiss et al. proposed an algorithm for molecularly stratifying urothelial carcinoma of the bladder patients for HER2 targeted therapy, taking into account gene expression, amplification, polysomy, and somatic mutation [17]. However, we still do not know if these therapies should be proposed to HER2 protein overexpressing or to HER2 FISH-positive urothelial carcinoma of the bladder. As low level polysomy is a frequent event in urothelial carcinoma of the bladder, further studies will be necessary to assess the benefit of anti-HER2 therapies in urothelial carcinoma of the bladder with HER2 polysomy. It will be mandatory also to track HER2 intratumoral heterogeneity to measure its impact on treated patients. Our study only focused on HER2 protein expression and HER2 gene status in locally advanced urothelial carcinoma of the bladder. We did not assess other potential drivers of advanced disease such non-amplifications HER2 alterations, FGFR, or PDL1 expression. Interestingly, intratumoral CD8-positive cells, high expression of PD1, and absence of PDL1 expression may predict favorable response to anti-HER2 therapies in HER2-positive breast cancers [43, 44]. Amplification of FGFR signaling may promote resistance to HER2 inhibition [45]. Further studies 
are required to identify predictive biomarkers to anti-HER2 therapies in urothelial carcinoma of the bladder.

\section{Conclusions}

Overall, our study suggests that HER2 evaluation should include an immunohistochemistry screening step with eligibility for FISH including moderate/strong and incomplete/complete membrane staining. To take into account the potential impact of spatial or temporal intratumoral heterogeneity, the evaluation should be performed on both tumor and lymph node, and for each histological variant observed. We hope that these recommendations will be helpful in assessing eligibility in future clinical trials.

Acknowledgements We thank Mrs Maryse Baia, Dr Cecile Charpy (Henri-Mondor hospital, Créteil), and Dr Julie Gonin (Tenon hospital, Paris) for technical advices.

\section{Compliance with ethical standards}

Conflict of interest The authors declare that they have no conflict of interest.

\section{References}

1. Wolff AC, Hammond ME, Schwartz JN, et al. Ame:rican Society of Clinical Oncology/College of American Pathologists guideline recommendations for human epidermal growth factor receptor 2 testing in breast cancer. J Clin Oncol. 2007;25:118-45.

2. Krüger S, Weitsch G, Büttner H, et al. Overexpression of c-erbB-2 oncoprotein in muscle-invasive bladder carcinoma: relationship with gene amplification, clinicopathological parameters and prognostic outcome. Int J Oncol. 2002;21:981-7.

3. Simon R, Atefy R, Wagner U, et al. HER-2 and TOP2A coamplification in urinary bladder cancer. Int $\mathrm{J}$ Cancer. 2003;107:764-72.

4. Latif Z, Watters AD, Dunn I, et al. HER2/neu overexpression in the development of muscle-invasive transitional cell carcinoma of the bladder. Br J Cancer. 2003;89:1305-9.

5. Coogan CL, Estrada CR, Kapur S, Bloom KJ. HER-2/neuprotein overexpression and gene amplification in human transitional cell carcinoma of the bladder. Urology. 2004;63:786-90.

6. de Pinieux G, Colin D, Vincent-Salomon A, et al. Confrontation of immunohistochemistry and fluorescent in situ hybridization for the assessment of HER-2/neu (c-erbb-2) status in urothelial carcinoma. Virchows Arch. 2004;444:415-9.

7. Latif Z, Watters AD, Dunn I, et al. HER2/neu gene amplification and protein overexpression in G3pT2 transitional cell carcinoma of the bladder: a role for anti-HER2 therapy? Eur J Cancer. 2004;40:56-63.

8. Hauser-Kronberger C, Peham K, Grall J, et al. Novel approach of human epidermal growth factor receptor 2 detection in noninvasive and invasive transitional cell carcinoma of the bladder. $\mathbf{J}$ Urol. 2006;175:875-80.

9. Caner V, Turk NS, Duzcan F, et al. No strong association between HER-2/neu protein overexpression and gene amplification in highgrade invasive urothelial carcinomas. Pathol Oncol Res. 2008;14:261-6
10. Matsubara H, Yamada Y, Naruse K, et al. Potential for HER-2/ neu molecular targeted therapy for invasive bladder carcinoma: comparative study of immunohistochemistry and fluorescent in situ hybridization. Oncol Rep. 2008;19:57-63.

11. Hansel DE, Swain E, Dreicer R, Tubbs RR. HER2 overexpression and amplification in urothelial carcinoma of the bladder is associated with MYC coamplification in a subset of cases. Am J Clin Pathol. 2008;130:274-81.

12. Laé M, Couturier J, Oudard S, et al. Assessing HER2 gene amplification as a potential target for therapy in invasive urothelial bladder cancer with a standardized methodology: results in 1005 patients. Ann Oncol. 2010;21:815-9.

13. Fleischmann A, Rotzer D, Seiler R, et al. Her2 amplification is significantly more frequent in lymph node metastases from urothelial bladder cancer than in the primary tumours. Eur Urol. 2011;60:350-7.

14. Cancer Genome Atlas Research Network. Comprehensive molecular characterization of urothelial bladder carcinoma. Nature. 2014;507:315-22.

15. Bellmunt J, Werner L, Bamias A, et al. HER2 as a target in invasive urothelial carcinoma. Cancer Med. 2015;4:844-52.

16. Nedjadi T, Al-Maghrabi J, Assidi M, et al. Prognostic value of HER2 status in bladder transitional cell carcinoma revealed by both IHC and BDISH techniques. BMC Cancer. 2016;16:653.

17. Kiss B, Wyatt AW, Douglas J, et al. Her2 alterations in muscleinvasive bladder cancer: patient selection beyond protein expression for targeted therapy. Sci Rep. 2017;7:42713 https://doi.org/ $10.1038 /$ srep42713

18. Oudard S, Culine S, Vano Y, et al. Multicenter randomized phase II trial of gemcitabine + platinum with or without Trastuzumab in advanced or metastatic urothelial carcinoma overexpressing HER2. Eur J Cancer. 2015;51:45-54.

19. Hofmann M, Stoss O, Shi D, et al. Assessment of a HER2 scoring system for gastric cancer: results from a validation study. Histopathology. 2008;52:797-805.

20. Ching CB, Amin MB, Tubbs RR, et al. HER2 gene amplification occurs frequently in the micropapillary variant of urothelial carcinoma: analysis by dual-color in situ hybridization. Mod Pathol. 2011;24:1111-9.

21. Schneider SA, Sukov WR, Frank I, et al. Outcome of patients with micropapillary urothelial carcinoma following radical cystectomy: ERBB2 (HER2) amplification identifies patients with poor outcome. Mod Pathol. 2014;27:758-64.

22. Tschui J, Vassella E, Bandi N, et al. Morphological and molecular characteristics of HER2 amplified urothelial bladder cancer. Virchows Arch. 2015;466:703-10.

23. Wolff AC, Hammond ME, Hicks DG, et al. Recommendations for human epidermal growth factor receptor 2 testing in breast cancer: American Society of Clinical Oncology/College of American Pathologists clinical practice guideline update. J Clin Oncol. 2013;31:3997-4013.

24. Hussain MH, MacVicar GR, Petrylak DP, et al. Trastuzumab, paclitaxel, carboplatin, and gemcitabine in advanced human epidermal growth factor receptor-2/neu-positive urothelial carcinoma: results of a multicenter phase II National Cancer Institute trial. J Clin Oncol. 2007;25:2218-24.

25. Choudhury NJ, Campanile A, Antic T, et al. Afatinib activity in platinum-refractory metastatic urothelial carcinoma in patients with ERBB alterations. J Clin Oncol. 2016;34:2165-71.

26. Powles T, Huddart RA, Elliott T. Phase III, double-blind, randomized trial that compared maintenance lapatinib versus placebo after first-line chemotherapy in patients with human epidermal growth factor receptor $1 / 2$-positive metastatic bladder cancer. J Clin Oncol. 2017;35:48-55.

27. Pouessel D, Bastuji-Garin S, Houédé N, et al. Adjuvant chemotherapy after radical cystectomy for urothelial bladder cancer: 
outcome and prognostic factors for survival in a French multicenter, contemporary cohort. Clin Genitourin Cancer. 2017;15: e45-e52.

28. Grignon DJ, Al-Ahmadie H, Algaba F, et al.. Tumours of the urinary tract. In: Moch H, Humphrey PA, Ulbright TM, Reuter VE, editors. WHO classification of tumors of the urinary system and male genital organs.. 4th ed. Lyon, France: International Agency for Research on Cancer; 2016. p. 77-133.

29. Humphrey PA, Moch H, Cubilla AL, et al. The 2016 WHO classification of tumors of the urinary system and male genital organs-part B: prostate and bladder tumors. Eur Urol. 2016;70:106-19.

30. Abd El-Latif A, Watts KE, Elson P, et al. The sensitivity of initial transurethral resection or biopsy of bladder tumor(s) for detecting bladder cancer variants on radical cystectomy. J Urol. 2013;189:1263-7.

31. Perez-Montiel D, Wakely PE, Hes O, et al. High grade urothelial carcinoma of the renal pelvis: clinicopathological study of 108 cases with emphasis on unusual morphological variants. Mod Pathol. 2006;19:494-503.

32. Guo CC, Dadhania V, Zhang L, et al. Gene expression profile of the clinically aggressive micropapillary variant of bladder cancer. Eur Urol. 2016;70:611-20.

33. Dadhania V, Zhang M, Zhang L, et al. Meta-analysis of the luminal and basal subtypes of bladder cancer and the identification of signature immunohistochemical markers for clinical use. EBio Med. 2016;12:105-17.

34. Ross JS, Wang K, Gay LM, et al. A high frequency of activating extracellular domain ERBB2(HER2) mutation in micropapillary urothelial carcinoma. Clin Cancer Res. 2014;20:68-75.

35. Kim B, Kim G, Song B, et al. HER2 protein overexpression and gene amplification in plasmacytoid urothelial carcinoma of the urinary bladder. Dis Markers. 2016;2016:8463731 https://doi.org/ $10.1155 / 2016 / 8463731$

36. Keck B, Ellmann C, Stoehr R, et al. Comparative genomic hybridization shows complex genomic changes of plasmacytoid urothelial carcinoma. Urol Oncol. 2014;32:1234-9.
37. Grillo F, Fassan M, Sarocchi F, Fiocca R, Mastracci L. HER2 heterogeneity in gastric/gastroesophageal cancers: from benchside to practice. World J Gastroenterol. 2016;22:5879-87.

38. Hou Y, Nitta H, Wei L, et al. HER2 intratumoral heterogeneity is independently associated with incomplete response to anti HER2 neoadjuvant chemotherapy in HER2 positive breast carcinoma. Breast Cancer Res Treat. 2017;166:447-57.

39. Hanna VW, Ruschoff J, Bilous M, et al. HER2 in situ hybridization in breast cancer: clinical implications of polysomy 17 and genetic heterogeneity. Mod Pathol. 2014;27:4-18.

40. Jimenez RE, Hussain M, Bianco FJ Jr, et al. Her-2/neu overexpression in muscle-invasive urothelial carcinoma of the bladder: prognostic significance and comparative analysis in primary and metastatic tumors. Clin Cancer Res. 2001;7:2440-7.

41. Lim TH, Lim AS, Thike AA, et al. Implications of the updated 2013 American Society of Clinical Oncology/College of American Pathologists guideline recommendations on human epidermal growth factor receptor 2 gene testing using immunohistochemistry and fluorescence in situ hybridization for breast cancer. Arch Pathol Lab Med. 2016;140:140-7.

42. Stoss OC, Scheel A, Nagelmeier I. Impact of updated HER2 testing guidelines in breast cancer re-evaluation of HERA trial fluorescence in situ hybridization data. Mod Pathol. 2015;28:1528-34.

43. Bianchini G, Pusztai L, Pienkowski T, et al. Immune modulation of pathologic complete response after neoadjuvant HER2-directed therapies in the NeoSphere trial. Ann Oncol. 2015;26:2429-36.

44. Hou Y, Nitta H, Wei L, et al. Evaluation of immune reaction and PD-L1 expression using multiplex immunohistochemistry in HER2 positive breast cancer: the association with response to anti HER2 neoadjuvant therapy. Clin Breast Cancer. 2017; https://doi. org/10.1016/j.clbc.2017.11.001

45. Hanker AB, Garrett JT, Estrada MV, et al. HER2 overexpressing breast cancers amplify FGFR signaling upon acquisition of resistance to dual therapeutic blockade of HER2. Clin Cancer Res. 2017;23:4323-34.

\section{Affiliations}

\section{Anissa Moktefi ${ }^{1}$ - Damien Pouessel ${ }^{2,3} \cdot$ Jing Liu $^{4,5,6} \cdot$ Nanor Sirab $^{2} \cdot$ Pascale Maille $^{2} \cdot$ Pascale Soyeux $^{2}$. Christiane Copie Bergman ${ }^{1} \cdot$ Marie Luce Auriault ${ }^{7} \cdot$ Dimitri Vordos $^{2,8} \cdot$ Alexandre de la Taille $^{2,8} \cdot$ Stéphane Culine ${ }^{9,10}$. Yves Allory ${ }^{2,11}$}

1 Assistance Publique-Hôpitaux de Paris (APHP), Pathology Department, Groupe Hospitalier Henri-Mondor/Albert Chenevier, Créteil F-94010, France

2 Département Hospitalo-Universitaire (DHU), Virus-ImmunitéCancer (VIC), Université Paris-Est-Créteil, (UPEC), Institut Mondor de Recherche Biomédicale (IMRB), Equipe 7 Translational Research of Genito-Urinary Oncogenesis, INSERM U 955, Créteil F-94010, France

3 Department of Medical Oncology, Institut Universitaire du Cancer Oncopole de Toulouse, Toulouse F-31053, France

4 Institut Curie, PSL Research University, CNRS, UMR144, Equipe Labellisée Ligue Contre le Cancer, Paris 75005, France

5 Sorbonne Universités, UPMC Université Paris 06, CNRS, UMR144, Paris 75005, France
6 Université Paris Sud, Université Paris-Saclay, Orsay 91405, France

7 Pathology Department, Centre hospitalier de La Rochelle, La Rochelle F-17000, France

8 Assistance Publique-Hôpitaux de Paris (APHP), Department of Urology, Groupe Hospitalier Henri-Mondor/Albert Chenevier, Créteil F-94010, France

9 Assistance Publique-Hôpitaux de Paris (APHP), Department of Medical Oncology, Hôpital Saint-Louis, Paris F-75010 France

10 Université Paris Diderot-Paris 7, Paris F-75010, France

11 Pathology Department, Institut Curie, Saint Cloud F-92210, France 